\title{
Supermassive and Intermediate-Mass Black Hole Growth at Galaxy Centers and Resulting Feedback using Cosmological Simulations
}

\author{
Paramita Barai* \\ Instituto de Astronomia, Geofísica e Ciências Atmosféricas - Universidade de São Paulo \\ (IAG-USP), Rua do Matão 1226, São Paulo, 05508-090, Brasil \\ E-mail: paramita.barai@iag.usp.br

\section{Elisabete M. de Gouveia Dal Pino} \\ Instituto de Astronomia, Geofísica e Ciências Atmosféricas - Universidade de São Paulo \\ (IAG-USP), Rua do Matão 1226, São Paulo, 05508-090, Brasil \\ E-mail: dalpino@iag.usp.br
}

\begin{abstract}
Accretion of matter onto central Black Holes (BHs) in galaxies liberates enormous amounts of feedback energy, which influence the formation and evolution of structures, affecting the environment from pc to Mpc scales. These BHs are usually Supermassive BHs (SMBHs: mass $\geq 10^{6} M_{\odot}$ ) existing at the centers of active galactic nuclei (AGN), which are widely observed through their multi-wavelength emission at all cosmic epochs. The SMBH energy output is often observed as powerful AGN outflows in a wide variety of forms. Relatively recently, Intermediate-Mass BHs (IMBHs: mass $=100-10^{6} M_{\odot}$ ) have started to be observed hosted in Dwarf Galaxy (DG) centers. Some of the central IMBHs in DGs show signatures of activity in the form of low-luminosity AGN.

We have performed Cosmological Hydrodynamical Simulations to probe SMBHs in high-z quasars [Barai et al. (2018)], and IMBHs in DGs [Barai \& de Gouveia Dal Pino (2019)]. Our simulations employ the 3D TreePM SPH code GADGET-3, and include metal cooling, star formation, chemical enrichment, stellar evolution, supernova feedback, AGN accretion and feedback. Analyzing the simulation output in post-processing, we investigate the growth of the first IMBHs, and the growth of the first SMBHs, their impact on star-formation, as well as their co-evolution with the respective host galaxies. We quantify the impact of SMBHs and IMBHs on their host galaxies, especially the effects on quenching star-formation. We also study the corresponding $\mathrm{BH}$ outflow properties.
\end{abstract}

International Conference on Black Holes as Cosmic Batteries: UHECRs and Multimessenger Astronomy BHCB2018

12-15 September, 2018

Foz du Iguazu, Brasil

${ }^{*}$ Speaker. 


\section{Introduction}

Active galactic nuclei (AGN) emit enormous amounts of energy powered by the accretion of gas onto their central supermassive black holes (SMBHs) (e.g., [Rees (1984)]). Feedback from AGN are believed to strongly influence the formation and evolution of galaxies (e.g., [Richstone et al. (1998), Barai (2008)]). A strong manifestation of AGN feedback are AGN outflows observed in a wide variety of forms (e.g., [Crenshaw, Kraemer \& George (2003), Feruglio et al. (2015)]).

Quasars are very powerful AGN existing more commonly at high- $z$ than in the local Universe (e.g., [Fan (2006)]). In the host galaxy of the quasar SDSS J1148+5251 at $z=6.4$, [Maiolino et al. (2012)] detected broad wings of the [CII] line tracing a massive outflow with velocities up to $\pm 1300 \mathrm{~km} / \mathrm{s}$. Follow-up by [Cicone et al. (2015)] revised the mass outflow rate lower limit to $1400 M_{\odot} / y r$. The physical mechanisms by which quasar outflows affect their host galaxies remain as open questions.

SMBHs of mass $\geq 10^{9} M_{\odot}$ are observed to be in place in luminous quasars by $z \sim 6$, when the Universe was less than 1 Gyr old (e.g., [Wu et al. (2015)]). It is difficult to understand how these early SMBHs formed over such short time-scales, and there are open issues with various plausible scenarios (e.g., [Matsumoto et al. (2015)]). AGN feedback should operate mostly in the negative form quenching star formation, as suggested by observations (e.g., [Schawinski et al. (2006)]), and simulations (e.g., [Scannapieco, Silk \& Bouwens (2005)]). At the same time, AGN feedback can occasionally be positive, by inducing star-formation, as have been shown in theoretical and numerical studies (e.g., [Zubovas et al. (2013)]), and observed in jet-induced star formation and radio-optical alignment (e.g., [Zinn et al. (2013)]).

Black holes are usually observed to belong to two populations: stellar-mass $\left(M_{\mathrm{BH}} \leq 10-\right.$ $\left.100 M_{\odot}\right)$ BHs, and supermassive $\left(M_{\mathrm{BH}} \geq 10^{6} M_{\odot}\right)$ BHs. By natural extension, there should be a population of Intermediate-Mass Black Holes (IMBHs: with mass between $100-10^{6} M_{\odot}$ ) in the Universe. Analogous to SMBHs producing AGN feedback, the IMBHs should also have feedback.

AGN feedback mechanism has recently started to been observed in low-mass galaxies. Investigating the presence of AGN in nearby dwarf galaxies using mid-infrared emission, [Marleau et al. (2017)] identified 303 candidates, of which $91 \%$ were subsequently confirmed as AGN by other methods. The stellar masses of these galaxies are estimated to be between $10^{6}-10^{9} M_{\odot}$; and the black hole masses in the range $10^{3}-10^{6} M_{\odot}$. [Penny et al. (2017)] presented observational evidence for AGN feedback in a sample of 69 quenched low-mass galaxies $\left(M_{\star}<4 \times 10^{9} M_{\odot}\right)$; including 6 galaxies showing signatures of an active AGN preventing ongoing star-formation.

The concordance $\Lambda \mathrm{CDM}$ cosmological scenario of galaxy formation presents multiple challenges in the dwarf galaxy mass range: e.g. core versus cusp density profile, number of DGs. Recently [Silk (2017)] made an exciting claim that the presence of IMBHs at the centers of essentially all old Dwarf Galaxies (DGs) can potentially solve the problems. Early feedback from these IMBHs output energy and affect the host gas-rich DGs at $z=5-8$. This early feedback can quench star-formation, reduce the number of DGs, and impact the density profile at DG centers.

In this work we present results of the growth and feedback of SMBHs in AGN, that of and IMBHs in DGs. We focus on negative BH feedback effects where star-formation is quenched. We performed zoomed-in cosmological hydrodynamical simulations of quasar-host galaxies at $z \geq 6$ to study their outflows (details in [Barai et al. (2018)]). Our goals are to investigate the impact of AGN outflows on host galaxies in the early Universe, and compute the simulated outflow properties; 
for which both observations and theoretical studies are scarce. We also performed cosmological hydrodynamical simulations of periodic comoving $(2 M p c)^{3}$ volumes, starting from $z=100$, to study IMBHs (details in [Barai \& de Gouveia Dal Pino (2019)]). Here, we investigate the scenario that IMBHs are present at the centers of all dwarf galaxies. Our goals are to test if IMBHs would grow at DG centers, and quantify the impact on star formation.

\section{Numerical Method and Simulations}

The initial conditions at $z=100$ are generated using the MUSIC ${ }^{1}$ software [Hahn \& Abel (2011)]. We use a modified version of the TreePM (particle mesh) - SPH (smoothed particle hydrodynamics) code GADGET-3 ([Springel (2005)]) to perform our cosmological hydrodynamical simulations. Radiative cooling and heating is incorporated from [Wiersma, Schaye \& Smith (2009)]. Eleven element species ( $\mathrm{H}, \mathrm{He}, \mathrm{C}, \mathrm{Ca}, \mathrm{O}, \mathrm{N}, \mathrm{Ne}, \mathrm{Mg}, \mathrm{S}, \mathrm{Si}, \mathrm{Fe})$ are tracked. Star-formation is implemented following the multiphase effective sub-resolution model by [Springel \& Hernquist (2003)], and chemical enrichment from [Tornatore et al. (2007)].

BHs are collisionless sink particles (of mass $M_{\mathrm{BH}}$ ) in our simulations. A BH (of initial mass $\left.M_{\mathrm{BHseed}}\right)$ is seeded at the center of each galaxy more massive than a total mass $M_{\text {HaloMin }}$, which does not contain a BH already. We test different values of minimum halo mass and seed BH mass in the range: $M_{\mathrm{HaloMin}}=\left(10^{6}-10^{7}\right) M_{\odot}$, and $M_{\mathrm{BHseed}}=\left(10^{2}-10^{3}\right) M_{\odot}$. The sub-resolution prescriptions for gas accretion onto BHs and kinetic feedback are adopted from [Barai et al. (2014), Barai et al. (2016)]. The halo mass $\left(M_{\text {halo }}\right)$ of a galaxy, and its virial radius in comoving coordinates $\left(R_{200}\right)$, are related such that $R_{200}$ encloses a density 200 times the mean comoving matter density of the Universe:

$$
M_{\text {halo }}=\frac{4 \pi}{3} R_{200}^{3}\left(200 \rho_{\text {crit }} \Omega_{M, 0}\right),
$$

where $\rho_{\text {crit }}=3 H_{0}^{2} /(8 \pi G)$ is the present critical density.

We execute a series of 4 Zoomed-In cosmological hydrodynamical simulations, with characteristics listed in Table 1. All the 4 runs incorporate metal cooling, chemical enrichment, SF and SN feedback. The first run has no AGN included, while the latter three explore different AGN feedback models. To create the zoom-in region, first a dark-matter (DM) only low-resolution simulation is carried out of a $(500 \mathrm{Mpc})^{3}$ comoving volume, using $256^{3} \mathrm{DM}$ particles, from $z=100 \mathrm{up}$ to $z=6$. Halos are identified within it using the Friends-of-Friends (FOF) algorithm. We select the most-massive halo at $z=6$, of a total mass $M_{\text {halo }}=4.4 \times 10^{12} M_{\odot}$, and a virial radius $R_{200} \simeq 511$ kpc comoving. We select the DM particles around it, inside a cubic box of side $2 R_{200}$. These DM particles are tracked back to our initial condition at $z=100$, and the Lagrangian region occupied by them is determined. The Lagrangian region (volume of $\left.(5.21 \mathrm{Mpc})^{3}\right)$ is populated with particles of higher resolution: DM and baryons, going from 8 to 13 levels of refinement. Finally, we restart the zoom-in cosmological simulation. The high-resolution particle masses are: $m_{\mathrm{DM}}=7.54 \times 10^{6} M_{\odot}$, and $m_{\mathrm{gas}}=1.41 \times 10^{6} M_{\odot}$. We employ $L_{\mathrm{soft}}=1 / h \mathrm{kpc}$ comoving as the Plummer-equivalent softening length for gravitational forces, for these high-resolution particles.

We perform cosmological hydrodynamical simulations of small-sized boxes with periodic boundary conditions, to probe dwarf galaxies at high redshifts. We execute a series of $10 \mathrm{sim}$ -

\footnotetext{
${ }^{1}$ MUSIC - Multi-scale Initial Conditions for Cosmological Simulations: https://bitbucket.org/ohahn/music
} 
Table 1: Zoomed-In Cosmological Hydrodynamical Simulations (for SMBHs)

\begin{tabular}{ccccc}
\hline $\begin{array}{c}\text { Run } \\
\text { name }\end{array}$ & $\begin{array}{c}\text { AGN feedback } \\
\text { algorithm }\end{array}$ & $\begin{array}{c}\text { Reposition of BH } \\
\text { to potential-minimum }\end{array}$ & $\begin{array}{c}\text { Geometry of region where } \\
\text { feedback is distributed }\end{array}$ & $\begin{array}{c}\text { Half opening angle } \\
\text { of effective cone }\end{array}$ \\
\hline noAGN & No BH & - & - & - \\
AGNoffset & Kinetic & No & Bi-Cone & $45^{\circ}$ \\
AGNcone & Kinetic & Yes & Bi-Cone & $45^{\circ}$ \\
AGNsphere & Kinetic & Yes & Sphere & $90^{\circ}$ \\
\hline
\end{tabular}

Table 2: Periodic-Box Cosmological Hydrodynamical Simulations (for IMBHs)

\begin{tabular}{ccccc}
\hline $\begin{array}{c}\text { Run } \\
\text { name }\end{array}$ & $\begin{array}{c}\text { BH } \\
\text { present }\end{array}$ & $\begin{array}{c}\text { Min. Halo Mass for BH Seeding, } \\
M_{\text {HaloMin }}\left[M_{\odot}\right]\end{array}$ & $\begin{array}{c}\text { Seed BH Mass, } \\
M_{\text {BHseed }}\left[M_{\odot}\right]\end{array}$ & $\begin{array}{c}\text { BH kinetic feedback } \\
\text { kick velocity } v_{w}(\mathrm{~km} / \mathrm{s})\end{array}$ \\
\hline SN & No & - & - & - \\
$B H s 2 h 1 e 6$ & Yes & $h^{-1} \times 10^{6}$ & $10^{2}$ & 2000 \\
$B H s 2 h 7 e 7$ & Yes & $5 h^{-1} \times 10^{7}$ & $10^{2}$ & 2000 \\
$B H s 3 h 1 e 7$ & Yes & $1 \times 10^{7}$ & $10^{3}$ & 2000 \\
$B H s 3 h 2 e 7$ & Yes & $2 \times 10^{7}$ & $10^{3}$ & 2000 \\
$B H s 3 h 3 e 7$ & Yes & $3 \times 10^{7}$ & $10^{3}$ & 2000 \\
$B H s 3 h 4 e 7$ & Yes & $4 \times 10^{7}$ & $10^{3}$ & 2000 \\
$B H s 3 h 4 e 7 v 5$ & Yes & $4 \times 10^{7}$ & $10^{3}$ & 5000 \\
$B H s 3 h 5 e 7$ & Yes & $5 \times 10^{7}$ & $10^{3}$ & 2000 \\
$B H s 4 h 4 e 7$ & Yes & $4 \times 10^{7}$ & $10^{4}$ & 2000 \\
\hline
\end{tabular}

ulations, with characteristics listed in Table 2. The size of the cubic cosmological volume is $\left(2 h^{-1} \mathrm{Mpc}\right)^{3}$ comoving, and we use $256^{3}$ dark matter and $256^{3}$ gas particles. The dark matter particle mass is $m_{\mathrm{DM}}=3.44 \times 10^{4} h^{-1} M_{\odot}$, and the gas particle mass is $m_{\text {gas }}=6.43 \times 10^{3} h^{-1} M_{\odot}$. The gravitational softening length is set to $L_{\mathrm{soft}}=0.1 h^{-1} \mathrm{kpc}$ comoving. Black holes of mass $1000 M_{\odot}$ are seeded inside halos when they reach a mass of $10^{7} M_{\odot}$. The black holes grow by accretion of gas from their surroundings and by merger with other black holes, and consequently eject feedback energy.

\section{Results and Discussion}

\subsection{Black Hole Accretion and Growth}

We find that first BHs are seeded at different cosmic times depending on the value of minimum

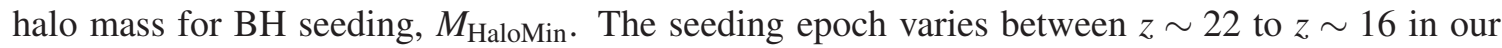
periodic-box cosmological simulations, when the first halos reach $M_{\text {halo }}=h^{-1} \times 10^{6} M_{\odot}$ to $M_{\text {halo }}=$ $5 \times 10^{7} M_{\odot}$. The redshift evolution of the most-massive BH mass in these periodic-box simulation runs is plotted in Fig. 1 - left panel. Each BH starts from an initial seed of $M_{\mathrm{BH}}=10^{2} M_{\odot}$ in the runs named $B H s 2^{*}, 10^{3} M_{\odot}$ in the runs named $B H s 3^{*}$, and $10^{4} M_{\odot}$ in the runs named $B H s 4^{*}$. The subsequent mass growth is due to merger with other BHs (revealed as vertical rises in $M_{\mathrm{BH}}$ ), and gas accretion (visualized as the positive-sloped regions of the $M_{\mathrm{BH}}$ versus $z$ curve). The final 

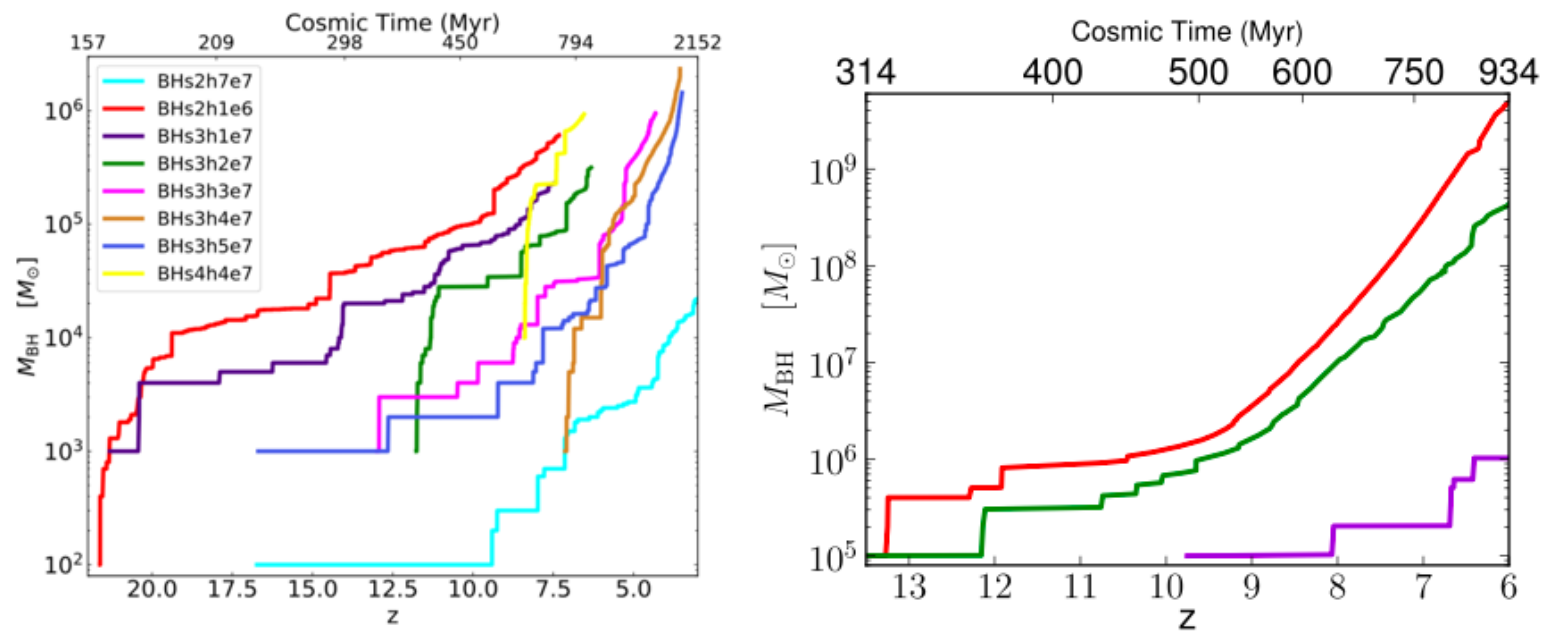

Figure 1: BH mass growth with redshift of the most-massive BH in each run. The different colours discriminate the various runs. Left panel: Periodic-Box cosmological simulations showing growth of IMBHs. (Figure modified from [Barai \& de Gouveia Dal Pino (2019)]). Right panel: Zoomed-In cosmological simulations showing growth of SMBHs. (Figure modified from [Barai et al. (2018)]).

properties reached depends on the simulation. The most-massive $\mathrm{BH}$, considering all the runs, has grown to $M_{\mathrm{BH}}=2 \times 10^{6} M_{\odot}$ at $z=5$ in run $B H s 3 h 4 e 7$ (brown curve in Fig. 1 - left panel).

The redshift evolution of the most-massive BH mass in the three AGN runs of the zoomed-in cosmological simulations is plotted in Fig. 1 - right panel. Each BH starts as a seed of $M_{\mathrm{BH}}=$ $10^{5} M_{\odot}$, at $z \sim 14$ in the runs AGNcone and AGNsphere $(z \sim 10$ in AGNoffset). The subsequent growth is due to merger with other BHs and gas accretion. The dominant mode of BH growth occurs over the redshifts $z=9-6$ in runs AGNcone and AGNsphere, corresponding to Eddingtonlimited gas accretion where Eddington ratio $=1$. The $\dot{M}_{\mathrm{BH}}$ has a power-law increase, and the $\mathrm{BH}$ mass increases by a factor $\sim 10^{3}$. The final properties reached at $z=6$ depends on the simulation; e.g. $M_{\mathrm{BH}}=4 \times 10^{9} M_{\odot}$ and $\dot{M}_{\mathrm{BH}}=100 M_{\odot} / \mathrm{yr}$ in run AGNcone (red curve). There is variability of the $\dot{M}_{\mathrm{BH}}$, whereby it fluctuates by a factor of up to 100 . The BH grows 10 times more massive at $z=6$ in the AGNcone case than in the AGNsphere run. This is because more gas can inflow along the perpendicular direction to the bi-cone, and accrete onto the $\mathrm{BH}$.

\subsection{Star Formation}

Stars form in the simulation volume from cold dense gas. The Star Formation Rate Density (SFRD in units of $M_{\odot} y r^{-1} M p c^{-3}$, counting stars forming in the whole simulation box) versus redshift of the periodic-box cosmological simulation runs is displayed in Fig. 2 - left panel. The SFRD rises with time in the $S N$ run (blue curve in Fig. 2 - left panel) initially from $z \sim 15$, reaches a peak at $z \sim 4$ (the peak epoch of star-formation activity in the Universe), and decreases subsequently over $z \sim 4-2$. The presence of a BH quenches star formation by accreting some gas in, ejecting some gas out of the halo as outflows, and/or heating the gas. The models suppress SF substantially from $z \sim 8$ onwards, when the BHs have grown massive. We find that BHs need to grow to $M_{\mathrm{BH}}>$ $10^{5} M_{\odot}$, in order to suppress star-formation, even in these dwarf galaxies. BH feedback causes a reduction of SFR up to 5 times in the different runs. 

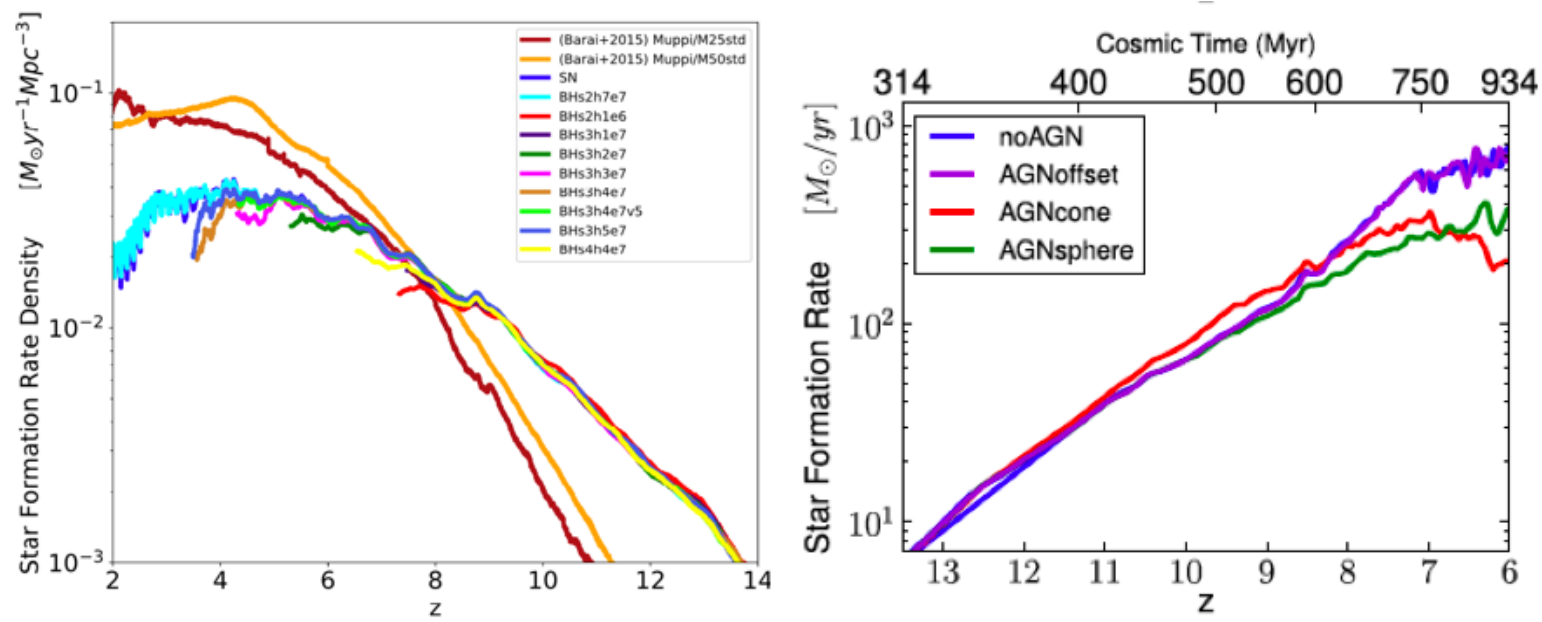

Figure 2: Left panel: Total star formation rate density (in $M_{\odot} y r^{-1} M p c^{-3}$, total SFR integrated over simulation volume) as a function of redshift, in the Periodic-Box cosmological simulations. (Figure modified from [Barai \& de Gouveia Dal Pino (2019)]). Right panel: Sum total star formation rate (in $M_{\odot} y r^{-1}$ ) as a function of redshift, in the Zoomed-In cosmological simulations. (Figure modified from [Barai et al. (2018)]).

The red curve (run $B H s 2 h l e 6$ ) already quenches SF as early as $z \sim 8$. This is because the BH has already grown to $M_{\mathrm{BH}} \sim 5 \times 10^{5} M_{\odot}$ at that epoch, more massive than all the other runs. As another example, the brown (run BHs3h4e7) and royal-blue (run BHs3h5e7) curves quench $\mathrm{SF}$ from $z \sim 4.5$ to $z \sim 3.5$. This is the epoch when the BH masses in these runs increase from $M_{\mathrm{BH}}=10^{5} M_{\odot}$ to $M_{\mathrm{BH}}=10^{6} M_{\odot}$ (as can be seen from Fig. 1 - left panel).

The star formation rate (total SFR in the simulation box) versus redshift of the four zoomed-in cosmological simulations is displayed in Fig. 2 - right panel. The SFR rises with time in all the runs initially, and continues to increase in the noAGN case without a BH. The SFR in run AGNoffset is almost similar to that in the run noAGN, because the BHs are too small there to generate enough feedback. A similar outcome happens in the runs AGNcone and AGNsphere at $z \geq 8$, when the BHs are too small.

The models suppress SF substantially from $z \sim 8$ onwards, when the BHs have grown massive and generate larger feedback energy. Thus, we find that BHs need to grow to $M_{\mathrm{BH}}>10^{7} M_{\odot}$, in order to suppress star-formation, even in massive galaxies ( of $M_{\star}=4 \times 10^{10} M_{\odot}$, and specific-SFR $=5 \times 10^{-9} \mathrm{yr}^{-1}$ ). BH feedback causes a reduction of SFR up to 4 times at $z=6$ : from $800 M_{\odot} / \mathrm{yr}$ in the $n o A G N$ run, to $200 M_{\odot} / \mathrm{yr}$ in run AGNcone, and $350 M_{\odot} / \mathrm{yr}$ in run AGNsphere.

\subsection{Large Scale Environment of IMBHs}

The gas morphology in our periodic cosmological simulation box, or the large scale structures, is plotted in Fig. 3. It displays the projected gas kinematics in the whole $\left(2000 h^{-1} \mathrm{kpc}\right)^{3}$ comoving volume at $z=4$, for two periodic-box simulations: $S N$ (left column) and $B H s 3 h 4 e 7 v 2$ (right column). The overdensity (i.e., the ratio between the gas density and the cosmological mean baryon density in the Universe) and temperature of the gas are plotted in the two rows from the top. The black circles in the top row depict the virial radius $R_{200}$ (defined in Eq. (2.1)) of dwarf galaxies 


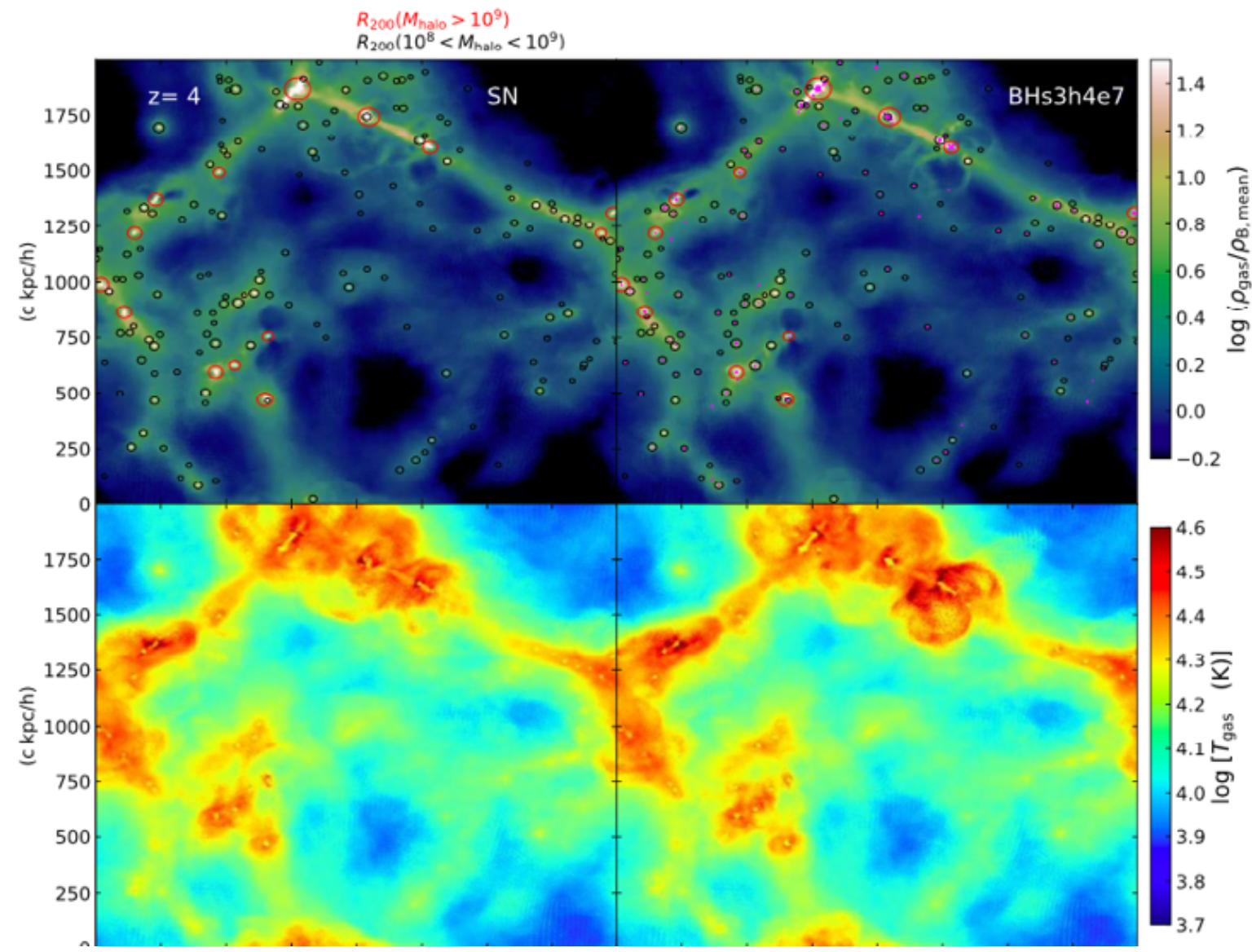

Figure 3: Projected gas kinematics in the whole $\left(2000 h^{-1} \mathrm{kpc}\right)^{3}$ periodic cosmological simulation box at $z=$ 4. The two rows present the gas overdensity (top row), and gas temperature (bottom row). The two columns are for different periodic-box simulations: $S N$ (left) and $B H s 3 h 4 e 7 v 2$ (right). The red circles in the top row depict the virial radius $R_{200}$ of galaxies in the mass range $M_{\text {halo }}>10^{9} M_{\odot}$, while the black circles show the $R_{200}$ of $10^{8}<M_{\text {halo }}<10^{9} M_{\odot}$ galaxies. The positions of BHs in run $B H s 3 h 4 e 7 v 2$ are indicated by the magenta cross-points in the top-right panel. (Figure modified from [Barai \& de Gouveia Dal Pino (2019)]).

with halo masses in the range $10^{8} \leq M_{\text {halo }} \leq 10^{9} M_{\odot}$. The red circles show the $R_{200}$ of relatively massive galaxies, those having higher halo masses $M_{\text {halo }}>10^{9} M_{\odot}$.

The spatial locations of the BHs within our $B H s 3 h 4 e 7 v 2$ simulation box can be visualized in the top-right panel of Fig. 3. Here the magenta cross-points designate $\mathrm{BH}$ positions, overplotted with the gas overdensity. In this run, BHs are seeded at the centres of galaxies with $M_{\text {HaloMin }}=$ $4 \times 10^{7} M_{\odot}$. Therefore all the red circles $\left(M_{\text {halo }}>10^{9} M_{\odot}\right.$ galaxies $)$ and most of the black circles ( $M_{\text {halo }}=10^{8}-10^{9} M_{\odot}$ galaxies) contain BHs at their centres.

The cosmological large-scale-structure filaments are visible in all the panels of both the runs. There are three Mpc-scale filaments: extending from east to north, from west to north, and from west to south. In addition, there is an overdense region running from the center of the box to the south-west. The filaments consist of dense (yellow and white regions in the top panels), and starforming gas. The massive galaxies (red circles) lie at the high-density intersections of the filaments, or in the filaments. 
In terms of temperature, the immediate vicinity of the dense filaments consists of hotter gas ( $T \sim 10^{4.6} \mathrm{~K}$, red regions in the bottom panels of Fig. 3), as compared to that in the low-density intergalactic medium and voids (yellow and blue regions). Several mechanisms play together to heat the gas to higher temperatures in the filament vicinity. There are global environmental processes like shock heating during galaxy mergers, and large-scale-structure formation, which are present in both the $S N$ and $B H s 3 h 4 e 7 v 2$ runs. Acting together there are local galactic processes like feedback driven by SN (present in both the columns), and BHs (present in the right column only), which heats the gas, and also often generate outflows.

As they accrete and grow, the BHs provide feedback energy (according to the prescription described in [Barai \& de Gouveia Dal Pino (2019)]), which may drive gas outflows. High-velocity gas ejected by central BH feedback propagates radially outward, and shocks with the surrounding slower-moving gas, creating bubble-like gas outflows. Our simulation $B H s 3 h 4 e 7 v 2$ shows the formation of BH feedback-induced outflows, as can be seen in Fig. 3 in the top-right half of the right column panels, around the most-massive $\mathrm{BH}$. The outflows are extended bipolar oval-shaped regions along the north-east to south-west direction, propagating to about $10 \times R_{200}$. The outflows consist of hot $\left(T>10^{4.6} \mathrm{~K}\right)$ - visible as red areas in the temperature map (bottom-right panel), and low-density gas (top-right panel).

\subsection{Quasar Outflow Morphology around SMBHs}

We find prominent gas outflows being developed in our simulated massive quasar-host galaxies, in the zoomed-in cosmological volumes. While the SMBHs output feedback energy, highvelocity gas propagates radially outward and shocks with the surrounding slower-moving gas. This creates bubble-like gas outflows originating from the central SMBH. The outflow morphology is plotted in Fig. 4, which displays the projected gas kinematics in a $\left(1000 h^{-1} \mathrm{kpc}\right)^{3}$ comoving volume in two zoom-in cosmological simulation runs, at $z=6$. The overdensity and temperature of the gas is plotted in the two columns.

In the noAGN run (top row), the outflows are weak $\left(v_{r}<300 \mathrm{~km} / \mathrm{s}\right)$, and bounded within $0.5 R_{200}$ as warm-hot $\left(T \sim 10^{6} \mathrm{~K}\right)$ halo gas. It is caused by $\mathrm{SN}$ feedback and galaxy merger shocks. Such a high-mass $\left(M_{\star} \sim 10^{11} M_{\odot}\right)$ galaxy cannot efficiently drive strong outflows with only SN feedback (e.g., [Benson et al. (2003), Talia et al. (2016)]).

The other two simulations (AGNcone and AGNsphere) show the formation of BH feedbackinduced powerful outflows, which are hot $\left(T \sim 10^{8} \mathrm{~K}\right)$ - visible as yellow and red areas in the temperature plot (bottom-right panel of Fig. 4), and consist of low-density, metal-enriched gas. The outflows are fastest $\left(v_{r}>2000 \mathrm{~km} / \mathrm{s}\right)$ in run AGNcone (bottom row, where the BHs become more-massive than the other runs), extended bipolar shaped, propagating to beyond the galaxy $R_{200}$ (black dashed circle). The outflows disrupt the cold dense filamentary gas inflows to the galaxy center, along the direction of outflow propagation. This quenches star formation, and halts the formation of nearby satellite galaxies. It is revealed by the disrupted clumps and lack of starforming dense filaments near the central galaxy in runs AGNcone and AGNsphere, as compared to run noAGN. The outflows transport metals away from star-forming regions, and enrich the surrounding circumgalactic medium out to $R_{200}$. Some inflows of cold, dense gas continue to occur perpendicular to the outflow direction. 


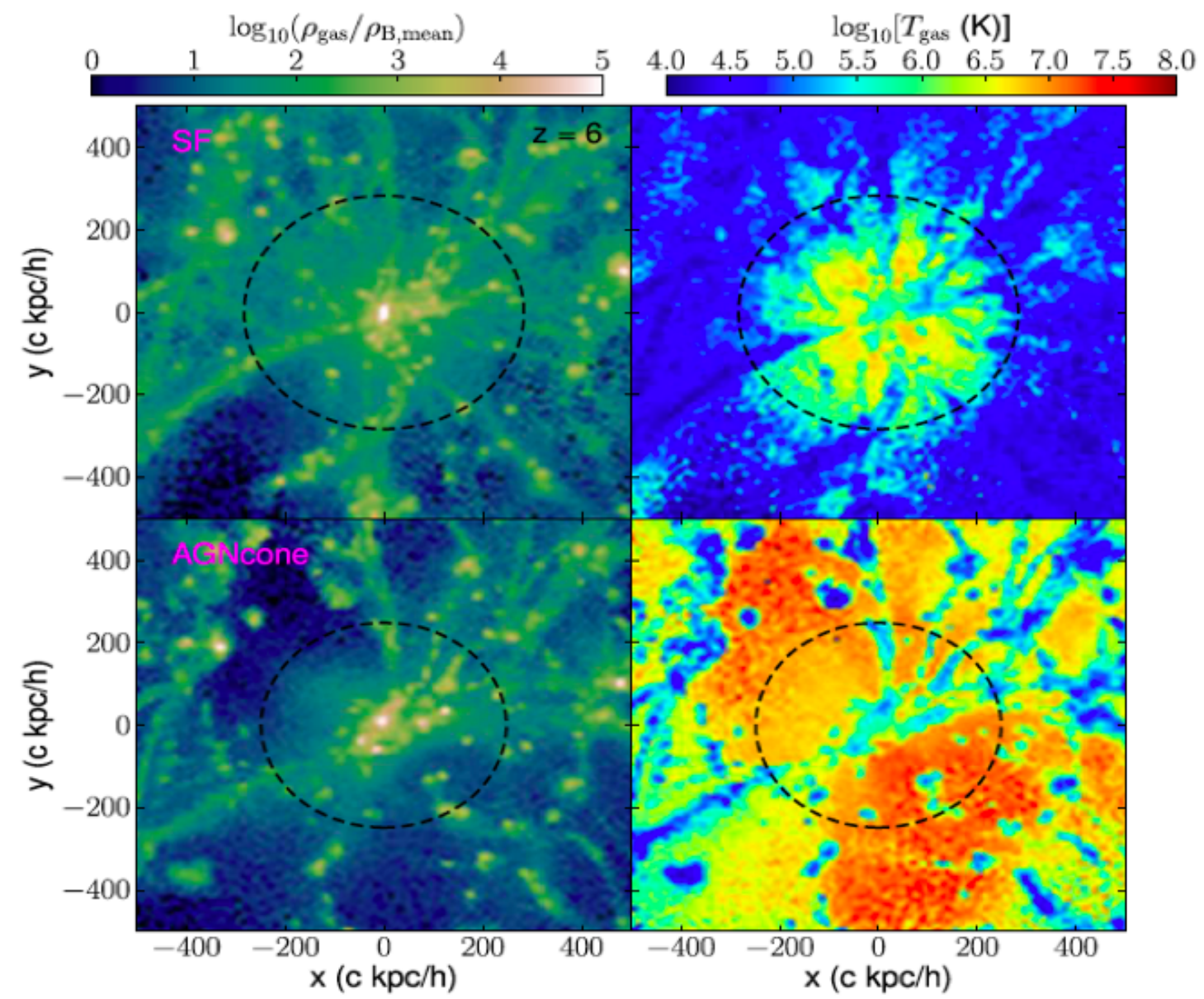

Figure 4: Projected gas kinematics in a $\left(1000 h^{-1} \mathrm{kpc}\right)^{3}$ comoving volume around the most-massive galaxy at $z=6$, in a Zoomed-In cosmological simulation. The two columns indicate gas overdensity (left column), and gas temperature (right column). The two rows are for different simulations: SF (top row), and AGNcone (bottom row). The black dashed circle depicts the galaxy virial radius $R_{200}$ in each case. (Figure modified from [Barai et al. (2018)]).

We find that the density increment, at the edges of the outflow shocks, remains below the SF threshold density parameter $(\$ 2)$ of our simulations. Therefore no new star-formation is triggered in our simulations by AGN feedback. This might be a numerical resolution effect, since our resolution (length scale of $1 \mathrm{kpc}$ comoving) is lower than those where positive feedback from AGN is simulated in galaxies (e.g., [Bieri et al. (2015)]).

\section{Conclusions}

Intermediate-mass black holes (mass between $100-10^{6} M_{\odot}$ ) have started to been observed at the centers of dwarf galaxies. We perform cosmological hydrodynamical simulations of $\left(2 h^{-1} \mathrm{Mpc}\right)^{3}$ comoving boxes with periodic boundary conditions, to probe dwarf galaxies and central IMBHs at high redshifts. We conclude that IMBHs at DG centers grow from $10^{2}-10^{3} M_{\odot}$ to $10^{5}-10^{6} M_{\odot}$ by $z \sim 4$ in a cosmological environment. These IMBHs in DGs can become the seeds of supermas- 
sive BHs (which grows to $M_{\mathrm{BH}} \sim 10^{9} M_{\odot}$ ) in massive galaxies. Star formation is quenched when the BHs have grown to $M_{\mathrm{BH}}>10^{5} M_{\odot}$. We find a positive correlation between the mass growth BHs and the quenching of SF. Our conclusions, based on numerical simulation results, support the phenomenological ideas made by [Silk (2017)]. IMBHs at the centers of dwarf galaxies can be a strong source of feedback to quench star-formation and generate outflows. At the same time, these IMBHs form the missing link between stellar-mass and supermassive BHs.

Gas accretion onto central supermassive black holes of active galaxies and resulting energy feedback, often manifested as AGN outflows, is an important component of galaxy evolution, whose details are still unknown especially at early cosmic epochs. We investigate outflows in quasar-host galaxies at $z \geq 6$ by performing cosmological hydrodynamical simulations. We simulate the $2 R_{200}$ region around a $2 \times 10^{12} M_{\odot}$ halo at $z=6$, inside a $(500 \mathrm{Mpc})^{3}$ comoving volume, using the zoomed-in technique. We find that, starting from $10^{5} M_{\odot}$ seeds BHs can grow to $10^{9} M_{\odot}$ in cosmological environments. During their growth, BHs accrete gas at the Eddington accretion rate over $z=9-6$, for 100s of Myr. At $z=6$, our most-massive BH has grown to $M_{\mathrm{BH}}=4 \times 10^{9} M_{\odot}$. Fast $\left(v_{r}>1000 \mathrm{~km} / \mathrm{s}\right)$, powerful $\left(\dot{M}_{\text {out }} \sim 2000 M_{\odot} / \mathrm{yr}\right)$ outflows of shock-heated low-density gas form at $z \sim 7$, and propagate up to hundreds kpc. Star-formation is quenched over $z=8-6$. The outflow mass is increased (and the inflow is reduced) by $\sim 20 \%$.

\section{Acknowledgements}

This work is supported by the Brazilian Funding Agencies FAPESP (grants 2016/01355-5, 2016/22183-8, and 2013/10559-5); and CNPq (grant 308643/2017-8).

\section{References}

[Barai (2008)] Barai, P. 2008, ApJ, 682, L17

[Barai et al. (2014)] Barai, P., Viel, M., Murante, G., Gaspari, M. \& Borgani, S. 2014, MNRAS, 437, 1456

[Barai et al. (2016)] Barai, P., Murante, G., Borgani, S., Gaspari, M., Granato, G. L., Monaco, P. \& Ragone-Figueroa, C. 2016, MNRAS, 461, 1548

[Barai et al. (2018)] Barai, P., Gallerani, S., Pallottini, A., Ferrara, A., Marconi, A., Cicone, C., Maiolino, R. \& Carniani, S. 2018, MNRAS, 473, 4003

[Barai \& de Gouveia Dal Pino (2019)] Barai, P. \& de Gouveia Dal Pino, E. M. 2018, submitted, eprint arXiv: 1807.04768

[Benson et al. (2003)] Benson, A. J., Bower, R. G., Frenk, C. S., Lacey, C. G., Baugh, C. M. \& Cole, S. 2003, ApJ, 599, 38

[Bieri et al. (2015)] Bieri, R., Dubois, Y., Silk, J. \& Mamon, G. A. 2015, ApJ, 812, L36

[Cicone et al. (2015)] Cicone, C. et al. 2015, A\&A, 574, A14

[Crenshaw, Kraemer \& George (2003)] Crenshaw, D. M., Kraemer, S. B., \& George, I. M. 2003, ARA\&A, 41,117

[Fan (2006)] Fan, X. 2006, NewAR, 50, 665

[Feruglio et al. (2015)] Feruglio, C. et al. 2015, A\&A, 583, A99 
[Hahn \& Abel (2011)] Hahn, O. \& Abel, T. 2011, MNRAS, 415, 2101

[Maiolino et al. (2012)] Maiolino, R. et al. 2012, MNRAS, 425, L66

[Marleau et al. (2017)] Marleau, F. R., Clancy, D., Habas, R. \& Bianconi, M. 2017, A\&A, 602, A28

[Matsumoto et al. (2015)] Matsumoto, T., Nakauchi, D., Ioka, K., Heger, A. \& Nakamura, T. 2015, ApJ, 810, 64

[Penny et al. (2017)] Penny, S. J. et al. 2017, submitted to MNRAS, eprint arXiv:1710.07568

[Rees (1984)] Rees, M. J. 1984, ARA\&A, 22, 471

[Richstone et al. (1998)] Richstone, D. et al. 1998, Nature, 395, A14

[Scannapieco, Silk \& Bouwens (2005)] Scannapieco, E., Silk, J. \& Bouwens, R. 2005, ApJ, 635, L13

[Schawinski et al. (2006)] Schawinski, K. et al. 2006, Nature, 442, 888

[Silk (2017)] Silk, J. 2017, ApJ, 839, L13

[Springel \& Hernquist (2003)] Springel, V. \& Hernquist, L. 2003, MNRAS, 339, 289

[Springel (2005)] Springel, V. 2005, MNRAS, 364, 1105

[Talia et al. (2016)] Talia, M. et al. 2016, submitted to MNRAS, eprint arXiv:1611.05884

[Tornatore et al. (2007)] Tornatore, L., Borgani, S., Dolag, K. \& Matteucci, F. 2007, MNRAS, 382, 1050

[Wiersma, Schaye \& Smith (2009)] Wiersma, R. P. C., Schaye, J. \& Smith, B. D. 2009, MNRAS, 393,99

[Wu et al. (2015)] Wu, X.-B. et al. 2015, Nature, 518, 512

[Zinn et al. (2013)] Zinn, P.-C., Middelberg, E., Norris, R. P. \& Dettmar, R.-J. 2013, ApJ, 774, 66

[Zubovas et al. (2013)] Zubovas, K., Nayakshin, S., King, A. \& Wilkinson, M. 2013, MNRAS, 433, 3079 